\title{
Médiévales
}

Langues, Textes, Histoire

61 | automne 2011

La chair des émotions

\section{Les communautés émotionnelles et le corps}

Emotional Communities and the Body

Barbara H. Rosenwein

\section{(2) OpenEdition}

Journals

Édition électronique

URL : https://journals.openedition.org/medievales/6278

DOI : $10.4000 /$ medievales.6278

ISSN : 1777-5892

Éditeur

Presses universitaires de Vincennes

Édition imprimée

Date de publication : 20 décembre 2011

Pagination : 55-75

ISBN : 978-2-84292-337-2

ISSN : 0751-2708

Référence électronique

Barbara H. Rosenwein, «Les communautés émotionnelles et le corps », Médiévales [En ligne], 61 |

automne 2011, mis en ligne le 17 janvier 2012, consulté le 24 avril 2022. URL : http://

journals.openedition.org/medievales/6278; DOI : https://doi.org/10.4000/medievales.6278 
Médiévales 61, automne 2011, p. 55-76

\title{
Barbara H. ROSENWEIN
}

\section{LES COMMUNAUTÉS ÉMOTIONNELLES ET LE CORPS}

\begin{abstract}
Le geste du corps et l'expression du visage doivent être observés avec attention, de sorte qu'à partir des choses extérieures on puisse rendre compte des choses intérieures. Car, puisque le visage est pour ainsi dire le sceau de l'âme, et sa forme visible, on peut par l'intermédiaire du visage accéder à la disposition interne.
\end{abstract}

Alain de Lille, Liber poenitentialis ${ }^{1}$.

Les émotions sont sans nul doute associées au corps. Cependant, comme j'essaierai de le montrer dans cet article, cette association était, et demeure, très changeante. Pour en débattre, je considérerai les émotions dans le contexte des communautés émotionnelles. Celles-ci correspondent à des groupes sociaux dans lesquels les individus sont animés par des intérêts, des valeurs et des styles émotionnels communs ou similaires. Les communautés émotionnelles développent ainsi diverses façons d'impliquer ou non le corps dans l'expression des émotions. Pour certaines d'entre elles, les émotions ne sont presque jamais

1. Alain de Lille, Liber poenitentialis, éd. J. Longère, 2 vol., Louvain, 1965, vol. II, p. 32 : «Considerandus est etiam corporis gestus, vel faciei habitus, ut per exteriora comprehendantur interiora, quia cum vultus sit quasi animi signaculum, et figura, per vultum potest perpendi quae est voluntas interna.» Ce conseil s'adresse aux prêtres en charge de la confession. Je souhaite remercier Damien Boquet et Piroska Nagy, ainsi que les participants au colloque «Habitus in Habitat I: Emotion and Motion» soutenu par le Zentrum für Literatur- und Kulturforschung Berlin, et également les «discutants» du séminaire organisé au Centre for Late Antique \& Medieval Studies at King's College London pour leurs commentaires avisés lors de la présentation d'une version préliminaire de cette étude. Enfin, je suis grandement redevable envers Damien Boquet pour la traduction française. 
associées à des manifestations somatiques. Pour d'autres, le lien est établi pour certaines catégories d'émotions seulement. Pour d'autres encore, presque toutes les émotions s'expriment au moyen du corps. Je m'intéresserai ici à trois communautés émotionnelles distinctes: la cour de Neustrie au viI siècle, celle qui réunit Thomas d'Aquin et ses disciples, et celle de Margery Kempe et de ses défenseurs. Nous verrons alors que l'affirmation tranchée d'Alain de Lille n'est pas toujours vérifiée.

\section{Physiologie émotionnelle, communautés émotionnelles}

Aujourd'hui encore, les conceptions d'Alain de Lille ont leurs partisans. Il suffit de se reporter par exemple aux travaux de Paul Ekman ${ }^{2}$ qui associent cinq expressions faciales discrètes à cinq "émotions élémentaires». Certains psychologues mettent l'accent sur le lien entre les gestes - y compris le fait de transpirer, de rougir, de pleurer et les manifestations du même genre - et les émotions ${ }^{3}$. Les neuropsychologues, quant à eux, soutiennent que les émotions sont produites, perçues et se reflètent à l'intérieur du cerveau ${ }^{4}$. D'autres scientifiques travaillent à partir de la neurochimie des émotions (dopamine, ocytocine, etc.) ${ }^{5}$. D'autres psychologues encore avancent que les métaphores mêmes qui sont couramment employées pour qualifier les émotions - par exemple «je fus saisi par une émotion»- sont la preuve de leur incorporation ${ }^{6}$.

2. Voir son étude originelle, désormais classique, P. Ekman et W. V. Friesen, «Constants across Cultures in the Face and Emotion», Journal of Personality and Social Psychology, 17/2 (1971), p. 124-129. Ses conclusions - à savoir que les expressions faciales de la joie, de la colère, de la tristesse, du dégoût, de la surprise et de la peur sont universelles et comprises universellement - sont aujourd'hui largement acceptées par les sciences psychologiques.

3. Sur les gestes, voir M. DE MEIJER, «The Contribution of General Features of Body Movement to the Attribution of Emotions », Journal of Nonverbal Behavior, 13/4 (1989), p. 247-68. Sur la transpiration, voir R. W. Levenson, «Blood, Sweat, and Fears: The Autonomic Architecture of Emotion», dans Emotions Inside Out : 130 Years after Darwin's The Expression of the Emotions in Man and Animals, New York, 2003 = Annales of the New York Academy of Sciences, 100 (2003), p. 348-366; J. T. CACIOPPO et ALII, «The Psychophysiology of Emotion», dans M. LewIS et J. M. Haviland-Jones éd., Handbook of Emotions, New York, 2000², p. 173-191.

4. Voir par exemple J. PANKSEPP, Affective Neuroscience: The Foundations of Human and Animal Emotions, New York, 1998, chap. 4. Pour une vue d'ensemble des relations entre les régions du cerveau, les mécanismes autonomiques et les émotions, voir H. D. CRitchley, «Neural Mechanisms of Autonomic, Affective, and Cognitive Integration», The Journal of Comparative Neurology, 493 (2005), p. 154-166.

5. H. E. Fisher, Why We Love: The Nature and Chemistry of Romantic Love, New York, 2004; C. S. CARTER, «Biological Perspectives on Social Attachment and Bonding», dans C. S. CARTER et ALI éd., Attachment and Bonding : A New Synthesis, Cambridge Mass., 2005, p. 85-100.

6. R. W. GibBs JR., Embodiment and Cognitive Science, Cambridge, 2006, en particulier le chap. 8 . 
Cependant, la recherche récente sur le cerveau suggère que la nature de cette incorporation n'est en aucune façon déterminée par la biologie. Le cerveau humain est hautement malléable et la conscience elle-même est tout autant structurée par la culture qu'elle engendre en retour cette culture. Dans un article suggestif, Robert Turner et Charles Whitehead ont présenté la synthèse d'une recherche qui s'appuie sur l'imagerie cérébrale pour montrer que la culture façonne «l'anatomie fonctionnelle et la microstructure du cerveau ${ }^{7} »$.

La démarche qui consiste à étudier les émotions immergées au sein de «communautés émotionnelles» fait partie de ces approches qui considèrent que les liens entre le corps et les émotions peuvent varier selon les cultures ${ }^{8}$. Certaines communautés émotionnelles donnent au corps toute sa place dans leur répertoire affectif, d'autres l'intègrent avec réserve - voire le rejettent tout à fait. Voyons dès lors comment se joue la partie à l'intérieur des trois communautés émotionnelles choisies ici comme terrain d'enquête.

\section{La cour de Neustrie : une cour «désincarnée»}

Commençons par une communauté émotionnelle qui évitait d'associer les émotions au corps. Au $\mathrm{VII}^{\mathrm{e}}$ siècle, en Neustrie, à la cour du roi mérovingien Clotaire II et de ses descendants, on pratiquait une extrême restriction émotionnelle ${ }^{9}$. Peu d'émotions étaient considérées favorablement, et leur incorporation était rarement légitimée - si ce n'est, comme nous le verrons, chez les femmes.

Les témoins qui nous donnent accès à cette communauté sont pour une large part des moines ou des évêques. Presque tous ont été éduqués à la cour. Ils ont continué, même après leur accession à l'épiscopat, à aller et venir entre le palais royal et leur cité. Jonas, qui fut moine puis abbé,évoluait à la marge de ce groupe. Il était lié à «l'évêque itinérant » saint Amand, une personnalité politique de premier plan qui évangélisa la région de Tournai au nom du roi ${ }^{10}$. Dès lors, la Vie de saint Colomban de Jonas est une source importante pour connaître les normes émotionnelles en vigueur à la cour de Neustrie.

7. R. Turner et C. Whitehead, «How Collective Representations Can Change the Structure of the Brain», Journal of Consciousness Studies, 15/10-11 (2008), p. 43-57.

8. Pour une réflexion approfondie sur cette dénomination, je me permets de renvoyer à B. H. RosenweIn, «Worrying about Emotions in History », American Historical Review, 107 (2002), p. 821-845 (en particulier p. 842-845) et EAD., Emotional Communities in the Early Middle Ages, Ithaca, 2006.

9. Pour une étude détaillée de cette communauté, voir B. H. Rosenwein, Emotional Communities..., chap. 5 .

10. Jonas de Bobbio, Vie de saint Colomban et de ses disciples, A. De Vogüé éd., Bégrolles-enMauges, 1988, p. 20-22; B. H. Rosenwein, Emotional Communities..., p. 134-135. 
Ici, comme pour les autres exemples de communautés émotionnelles que je considérerai par la suite, j'ai choisi de retenir un texte représentatif afin d'en explorer les ramifications rattachées à mon propos. Dans le cas présent, ce texte est une lettre de Didier, évêque de Cahors (630-655), adressée à l'évêque Dadon au début des années $640{ }^{11}$. Avant son accession à l'épiscopat, Didier avait assumé la charge de trésorier au service du roi Clotaire puis de son fils Dagobert ${ }^{12}$. Dadon, son correspondant, avait été le référendaire (une sorte de protochancelier) de Dagobert ${ }^{13}$. Le cours de sa vie fut décidé comme sous l'effet d'un charme: dans sa jeunesse, en effet, il avait été béni par le véhément et charismatique saint Colomban. Après avoir servi le roi à la cour, il devint évêque de Rouen et fut connu, après sa mort, sous le nom de saint Ouen. Dans la lettre, d'anciens hommes de cour sont nommés, rappelant ainsi le bon vieux temps d'une jeunesse passée au palais du roi :

Au pape Dadon, père apostolique saint et préféré, Didier, serviteur des serviteurs de Dieu. Tandis qu'un long moment s'est écoulé sans que nous puissions jouir de ta présence, aujourd'hui une joie (gratulatio) immense s'est présentée à mon esprit car, après un tel laps de temps, l'opportunité s'offre enfin à moi d'être dans une certaine mesure présent devant tes yeux par l'entremise d'une lettre. Ainsi, assuré d'avoir fait preuve avec humilité de mon devoir d'obéissance, je te soumets cette demande spéciale afin que tu acceptes et daignes toujours être celui qui me montra un amour unique (unico amore) dans la fleur de la jeunesse printanière, à savoir mon cher Dadon. Que cet amour (caritas) d'antan, inébranlable, qui exista entre nous, et avec celui qui t'est cher, ou plutôt qui nous est cher, Éloi, perdure, indivisible comme le fut notre fraternité. Puissions-nous par le soutien de nos prières mutuelles mériter de vivre réunis dans le palais céleste du très haut roi de la même manière que nous fûmes liés à la cour du prince terrestre. Et bien que j'aie perdu aujourd'hui deux frères de notre collège [Rustique et Syagrius, frères de sang de Didier], nous avons à leur place le vénérable Paul, et Sulpice, qui n'est pas moins remarquable par ses mérites. Ainsi, aussi haut que l'un d'entre nous s'élève, permettons-lui de gravir plus haut encore l'échelle du succès. D'ailleurs, je l'affirme, je suis plus assuré de pouvoir y parvenir par tes prières que par mes propres forces. Il suffit que tu acceptes de prier sans relâche et la piété de notre seigneur Jésus-Christ, je le crois, t’apportera ce que tu sollicites. Porte-toi bien, homme de Dieu, et souviens-toi de moi ${ }^{14}$.

11. Didier de Cahors, Epistula 1.11, D. Norberg éd., Stockholm, 1961 (Studia Latina Stockholmiensia, 6), p. 30. Lettre datée par D. Norberg de 641-646.

12. Voir H. Ebling, Prosopographie der Amtsträger des Merowingerreiches von Chlothar II. (613) bis Karl Martell (741), Munich, 1974, p. 126-127, n. 142.

13. Ibid., p. 124-125, n. 141; P. Fouracre et R. A. Gerberding, Late Merovingian France: History and Hagiography 640-720, Manchester, 1996, p. 136-152.

14. Didier De CAHORS, Epistula, 1.11, p. 30: «Sancto ac preferendo apostolico patre Dadone papae Desiderius servus servorum dei. Dum plurima tempora elabuntur, quod praesentiam vestram 
Ne nous laissons pas troubler par le titre de «pape» : c'est ainsi qu'en Gaule les évêques s'adressaient les uns aux autres au vII siècle. Intéressons-nous plutôt aux voies empruntées par Didier pour désincarner l'affection qu'il désire exprimer. La lettre a pour finalité de renouer le contact avec Dadon après une longue période de silence et de lui rappeler qu'ils ont été étroitement liés l'un à l'autre par le passé - ainsi qu'avec un autre homme, Éloi, lui aussi homme de cour qui, au moment où est rédigée la lettre, occupe la charge d'évêque de Noyon. La lettre en appelle à une association de prière informelle entre les trois hommes afin que leur amitié puisse se prolonger au-delà de cette vie terrestre. Elle assigne à deux autres personnes, Paul et Sulpice (eux aussi autrefois gens de cour et désormais évêques), un statut équivalent aux frères de sang de Didier récemment assassinés, signifiant par là que leur fraternité lui est également précieuse. En résumé, il s'agit d'une lettre d'amour.

Pour autant l'émotion incarnée est constamment tenue à distance. Le salut initial évite toute marque d'affection. Toutes les autres lettres de cette collection, qui en compte 37 , font de même, à une exception près. Il s'agit d'une épître de Bertegisèle, dont l'adresse fleurie qualifie Didier de «seigneur illustre et chéri par nous du plus grand amour (dilectione) ${ }^{15}$. Mais cette exception confirme la règle: Bertegisèle n'appartient aucunement à la communauté émotionnelle de la cour neustrienne. Il était abbé de Saint-Victor de Marseille et sa lettre reflète sans aucun doute des normes sensiblement différentes de celles qui prévalent pour Didier et ses compagnons.

Poursuivons la lecture de la lettre de Didier. Il manifeste sa joie d'être enfin en mesure de correspondre avec Dadon. Cependant il ne l'exprime pas au moyen d'une métaphore corporelle - du genre «je suis ivre de joie» - mais plutôt par une construction passive: «une grande joie s'est présentée à mon esprit». Ensuite, il demande à Dadon d'être cette même personne qui, autrefois dans sa jeunesse, lui manifestait un amour unique. Une fois encore, l'émotion

videre nequimus, nunc inmensa se gratulatio menti objecit, dum aliquatenus, vel post diutina intervalla, sese opportunitas praehibuit, qua vel pagellari offitio me vestris conspectibus praesentarem. Igitur, debito obsequio humiliter exhibeto, illud peculiarius peroro ut, quem quondam in ipso flore primevae juventutis unico mihi amore prebuisti, semper concedere digneris illum meum Dadonem. Maneat pristina inter nos atque illum tuum, immo nostrum Elegium inconvulsa caritas, indisjuncta, ut fuit quondam, fraternitas. Mutuis nos jubemus praecibus, ut, quemadmodum in aula terreni principis socii fuimus, ita in illo superni regis caelesti palacio simul vivere mereamur. Et licet de nostro collegio duos iam amiserim germanos, habemus pro his venerabilem Paulum nec minus praedicabilem meretis Sulpicium. Quisquis igitur nostrum quantum plus praevalet, tanto amplius profectuum grados conscendere elaboret. Ad haec autem, predico, plus me vestris orationibus quam meis viribus adtingere posse confido. Tantum est ut indesinenter vos orare dignetis, et pietas Domini nostri Jesu Christi, credo, praestabit, quod rogatis. Vale, vir Dei, et memento mei.»

15. Ibid., 2.2, p. 45: «Domino inlustri et a nobis summa dilectione colendo.» 
est objectivée comme quelque chose d'extérieur aux deux hommes, Didier et Dadon. Comme c'est le cas pour la joie de Didier, cette affection est présentée plus qu'elle n'est ressentie. Cet amour est rendu encore plus éthéré lorsqu'il est exprimé, dans la phrase suivante, par le mot caritas, puis lorsqu'il est associé à l'amitié d'Éloi et à l'espoir d'une fraternité prolongée dans l'au-delà. En outre, la mort des frères de Didier, pourtant soudaine et brutale, est signalée sans aucune passion: eux sont partis, mais Paul et Sulpice demeurent.

La plupart des textes produits par ce groupe neustrien, qu'ils proviennent de Didier ou de ses proches, reprennent ce ton sec, sans épaisseur charnelle. Pour autant, il existe des moments particulièrement parlants, dans ce contexte d'aridité générale, où les émotions sont incorporées. Dans une lettre adressée à l'abbesse Aspasie, Didier écrit qu'il fut «ébranlé» (motus) par ses larmes ${ }^{16}$. Ainsi, elle ne refrène pas son émotion incorporée, tandis que lui use d'un vocabulaire qui évoque le corps pour signifier la chose. Les femmes, dans cette communauté émotionnelle, pouvaient exprimer leurs émotions corporellement, et elles le faisaient. Mais au regard des normes masculines, elles étaient dans le même temps dépréciées parce qu'elles se comportaient ainsi. Il suffit de considérer la façon dont la mère de Colomban est présentée dans la Vita Columbani de Jonas lorsqu'elle apprend que son fils est sur le point de la quitter:

Sa mère, brisée par la douleur, l'implore de ne pas la quitter. Mais il lui dit: «N'as-tu pas entendu la parole: "Celui qui aime son père et sa mère plus que moi, celui-là n'est pas digne de moi ?" [Matt. 10,37]» Il demande à sa mère, qui lui fait obstacle, le corps étendu sur le pas de la porte, de le laisser partir. Poussant des cris de douleur, prostrée sur le pavement, elle refuse qu'il s'en aille. Il enjambe (transilit) sa mère et le seuil, et incite sa mère à se réjouir (se laetam habeat): elle ne le reverrait plus jamais en cette vie, mais il irait là où la voie du salut lui indiquerait le chemin ${ }^{17}$.

Sous la plume de Jonas, la mère de Colomban n'est pas «présentée» dans un état de tristesse, comme Didier est présenté dans un état de joie dans sa lettre, elle est frappée par le chagrin, comme si on lui assénait un coup de poing. (Le mot latin que je traduis ici par «frappée», stimulata, vient de stimulus qui peut désigner une pique utilisée pour conduire les animaux ou un instrument pour

16. Ibid., 1, 15, p. 37 : «Lacrimis tuis hactenus motus...»

17. Jonas, Vitae Columbani abbatis discipulorumque eius, 1.3, B. Krusch éd., Hanovre, 1902 (Monumenta Germaniae Historica, Scriptores rerum merovingicarum, 4), p. 69: «Materque eius dolore stimulata, precatur, ut se non relinquat. At ille: "Non", inquid audisti: "Qui amat patrem aut matrem plus quam me, non est me dignus ?" Obstanti matri et limitem ostii inherenti postulat, ut se ire sinat. Illa eiulans et pavimento prostrata, denegat se permissuram; ille limitem matremque transilit poscitque matri, se laetam habeat: illum numquam deinceps in hac vita visurum, sed, quocumque salutis via iter pandat, se progressurum.» 
torturer les personnes.) Elle barre le chemin à son fils, gémissante, puis tombe à terre. Tournons-nous maintenant vers le fils. Impassible devant ses marques d'affection, il enjambe son corps prostré avec un aplomb froid. Certes, il y a bien un mouvement ici, on peut en être sûr, mais il est le fruit du calcul, non de la passion. Puisque Colomban est le héros du récit, il fait figure de modèle de comportement. Nous avons affaire en la circonstance à une communauté émotionnelle qui reconnaît la potentialité de l'émotion à s'incorporer, mais qui salue en même temps la vertu de ceux qui dominent cette tendance.

\section{Les larmes de Thomas d'Aquin}

Le dominicain Thomas d'Aquin (1225-1274) appartient à deux communautés émotionnelles au moins: la première, large, est celle de son ordre religieux ; la seconde, qui est un sous-ensemble de la première, est formée par ses disciples. Ce second groupe comprend notamment Ptolémée de Lucques (mort vers 1326), Remigio de' Girolami (mort en 1319) et Guillaume de Tocco (mort en 1322/1323). Ceux-ci ont suivi l'enseignement du maître, ils l'ont accompagné dans ses voyages. Pour ce qui concerne Guillaume, il a même écrit sur son héros ${ }^{18}$. Leurs écrits à tous portent l'empreinte de la pensée de Thomas sur les émotions qui est longuement exposée dans sa Somme théologique ${ }^{19}$. Ce n'est pas le lieu ici de considérer les relations entre les théories des émotions et leur pratique, mais il est clair que si les émotions sont étroitement liées à la cognition, comme cela est communément admis aujourd'hui, alors elles seront façonnées en fonction de ce que les gens estiment vrai et important ${ }^{20}$.

Pour Thomas, les émotions sont liées aux puissances de l'âme, et l'âme elle-même est fermement arrimée au corps. Thomas suit le De anima d'Aristote quand il identifie les cinq puissances de l'âme: «végétative, sensitive, appétitive, motrice et intellective ${ }^{21}$. Les émotions (ou, pour utiliser le terme de Thomas, les

18. Sur les disciples de Thomas, voir I. Taurisano, «Discepoli e biografi di S. Tommaso », dans S. Tommaso d'Aquino O.P. Miscellanea storico-artistica, Rome, 1924, p. 111-186; sur Remigio en particulier, voir M. M. MulchaheY, «First the Bow Is Bent in Study...», Dominican Education before 1350, Toronto, 1998, en particulier p. 389-396; voir aussi EAD., «Education in Dante's Florence Revisited: Remigio de'Girolami and the Schools of Santa Maria Novella», dans R. B. Begley et J. W. Koterski éd., Medieval Education, New York, 2005, chap. 10.

19. S. Knuuttila, Emotions in Ancient and Medieval Philosophy, Oxford, 2004, p. 239, qualifie l'exposé de Thomas sur les passions dans la Summa theologiae de «traité médiéval le plus complet sur le sujet».

20. La revue Cognition and Emotion publie des articles qui traitent, entre autres choses, du «rôle des processus cognitifs dans le déclenchement, la régulation et l'expression de l'émotion» (http://www.tandf.co.uk/journals/pp/02699931.html).

21. Thomas D’Aquin, Summa theologiae, I, q. 78, a. 1, arg. 1. 
passions) relèvent toutes, à de rares exceptions près, de la puissance appétitive, laquelle se trouve en position médiane entre, d'une part, la puissance végétative (partagée avec les plantes) et la puissance sensitive (propre aussi aux animaux) et, d'autre part, les puissances supérieures, spécifiques aux hommes, aux anges et à Dieu. Thomas établit des subdivisions à l'intérieur de la puissance appétitive : l'une d'elles, «l'appétit intellectif», est à son tour divisée entre la volonté (voluntas) et le libre arbitre (liberum arbitrium) ${ }^{22}$. Les passions trouvent leur origine dans une autre subdivision, «l'appétit sensitif», qui peut être de deux espèces, concupiscible ou irascible ${ }^{23}$.

Ainsi, toutes les passions sont associées au corps et s'accompagnent de modifications somatiques tangibles, comme par exemple une contraction (produite par la peur) ou une expansion (un effet du plaisir) ${ }^{24}$. La peur glace les membres de ceux qui la ressentent, les empêchant de crier, et elle les fait trembler ${ }^{25}$. La tristesse de son côté se manifeste souvent par des larmes ${ }^{26}$.

Cependant les émotions peuvent transcender le corps si elles sont correctement orientées. Ici, l'amour est la passion déterminante. Thomas l'appelle «la racine première de toutes les émotions ${ }^{27}$. Elle est l'origine de toutes les émotions qui tendent vers le bien (même si parfois ce «bien» peut être identifié de façon impropre et irrationnelle). Même la haine, qui est l'opposé de l'amour, est déterminée dans un premier temps par l'orientation de l'amour, dans la mesure où elle vise n'importe quel objet opposé à l'objet de l'amour. L'amour est ainsi à l'origine de toute la chaîne des émotions: de l'amour découle le désir, du désir découle la joie. Ou bien, lorsque l'amour est contrarié, ce sont les émotions de l'irascible qui entrent en scène, à savoir les passions de l'espoir, de la peur, du désespoir et de la colère ${ }^{28}$.

Thomas identifie deux sortes d'amour. L'amour de concupiscence (amor concupiscentiae), le plus commun, vise le bien qu'on veut pour soi-même ou pour un autre. Quant à l'amour d'amitié (amor amicitiae), il s'oriente vers celui à qui nous voulons du bien ${ }^{29}$. Ces deux formes d'amour entraînent vers leur objet si puissamment qu'elles produisent trois effets extraordinaires: l'union, l'inhabitation mutuelle et l'extase (extasis ou raptus) ${ }^{30}$. Ces trois effets extirpent

22. Ibid., I, q. 83, a. 4.

23. Ibid., I, q. 81, a. 2 .

24. Ibid., I-II, q. 41, a. 1, co. Sur la contraction due à la peur, voir ibid., I-II, q. 41, a. 1, co.; sur l'expansion provoquée par le plaisir, voir I-II, q. 33, a. 1.

25. Ibid., I-II, q. 44, a. 1, ad 2.

26. Ibid., I-II, q. 32, a. 4.

27. Ibid., I-II, q. 46, a. 1: «Amor enim est prima radix omnium passionum».

28. Ibid., I-II, q. 23, a. 4, co.

29. Ibid., I-II, q. 26, a. 4.

30. Ibid., I-II, q. 28, considère les trois effets. 
dans une certaine mesure celui qui aime hors de lui-même, même si le corps est encore impliqué dans les deux premiers. En revanche, l'extase, en particulier la forme d'extase qui survient «selon la puissance intellective», arrache l'individu à lui-même et le projette au-delà de lui-même ${ }^{31}$. Ce processus ne se produit que de façon partielle dans «l'amour de concupiscence» où l'amour est encore dirigé vers soi. En revanche, il s'accomplit entièrement dans «l'amour d'amitié» où «l'affect porté à quelqu'un sort simplement hors de soi » ${ }^{32}$.

Le texte que j'ai retenu comme emblématique de la communauté émotionnelle de Thomas est extrait de l'Ystoria sancti Thome de Aquino de Guillaume de Tocco et provient d'une partie où il est question de la pureté de Thomas:

Sa prière était empreinte d'une dévotion qui dépassait la mesure. Ainsi, par la dévotion de son esprit, il s'élevait librement jusqu'à Dieu, comme s'il n'avait pas à supporter le poids contraire de sa chair. Il avait coutume de ne jamais exciter une émotion (motum) contraire à la raison, ainsi il contraignait son corps à être soumis à sa raison. Son humilité, sa conversion et sa pureté sont rendues manifestes non seulement par ce qui a déjà été dit [plus haut] mais aussi par ce qui va suivre et qui a été prouvé par un témoignage véridique.

Il avait une dévotion particulière pour le saint sacrement de l'autel. Parce qu'il lui avait été donné d'écrire à son propos des choses d'une grande profondeur [dans ses textes sur la fête du Corps du Christ], il avait également reçu le don de le célébrer avec une grande dévotion. En effet, il disait une messe chaque jour, sauf si une maladie l'en empêchait, et [chaque jour aussi] il entendait une autre messe, dite par un compagnon ou bien par quelqu'un d'autre, qu'il servait aussi souvent que possible. De plus, il était fréquent qu'il fût saisi (rapi) durant la messe par un sentiment de dévotion (divotionis affectu) si fort - lui qui était absorbé dans les mystères saints d'un tel sacrement et restauré par ses dons qu'il en était tout entier baigné de larmes. Ainsi, au couvent de Naples, le jour de la Passion du seigneur, il arriva qu'il célébrât la messe en grande dévotion tandis que de nombreux chevaliers étaient à proximité. Alors que la célébration des mystères sacrés commençait, il fut subitement absorbé par la profondeur

31. Ibid., I-II, q. 28, a. 3, co.: «Dicendum quod extasim pati aliquis dicitur, cum extra se ponitur. Quod quidem contingit et secundum vim apprehensivam, et secundum vim appetitivam.» («On dit de quelqu'un qu'il est en extase lorsqu'il est mis hors de soi. Cela peut arriver selon la puissance appréhensive et selon la puissance appétitive.») Que la vis apprehensiva puisse être comprise ici dans le sens de «puissance intellective» est suggéré ibid., I-II, q. 15, a. 1, arg. 3: «Sed assentire pertinet ad intellectum, qui est vis apprehensiva.» («Mais assentir se rapporte à l'intellect qui est la puissance appréhensive.») À la fin de I-II, q. 28, a. 3, co., Thomas conclut qu'une véritable extase n'est possible qu'au travers de l'intellect. Les notions d'extase chez Thomas sont présentées dans B. FAes de MottonI, «Excessus mentis, alienatio mentis, estasi, rapus nel medioevo», dans E. CANone éd., Per una storia del concetto di mente, Florence, 2005, p. 181-184.

32. Thomas D’Aquin, Summa theologiae, I-II, q. 18, a. 4, co.: « ... affectus alicuius simpliciter exit extra se». 
du sacrement comme s'il avait été associé aux mystères divins et subissait les châtiments du Christ homme. Cela se voyait au ravissement de son esprit (mentis abstractio) et au flot abondant de ses larmes. Après qu'il fut resté dans cet état un long moment, ses frères, remplis d'admiration, vinrent à lui et, le touchant pour l'inciter à poursuivre les saints mystères, ils le firent revenir de la profondeur des mystères où il semblait avoir été ravi (raptus) ${ }^{33}$.

Ce passage complexe laisse voir un Guillaume bataillant entre une conception stoïcienne et chrétienne des émotions qui montre son héros maintenu à l'écart de la sphère charnelle et une conception aristotélicienne où les émotions sont liées au corps. Guillaume concentre ses espoirs d'une pratique stoïcienne dans une phrase clé, lorsqu'il écrit que Thomas avait comme habitude de «ne jamais exciter une émotion (motum) contraire à la raison, ainsi il contraignait son corps à être soumis à sa raison». Thomas lui-même avait donné à Guillaume un bon argument pour penser la chose possible en affirmant, à propos de l'extase « selon la puissance intellective», qu'elle arrachait l'individu à soi et le projetait hors de lui-même, y compris hors de son corps. Or, on sait que l'extase est provoquée par l'amour, mais Thomas aimait-il ? Oui, dit Guillaume dans une anecdote située plus haut dans l'Ystoria où il montre Thomas repoussant les avances d'une très belle jeune fille (puellam pulcherrimam) au titre qu'il avait déjà une épouse (sponsa): la sagesse de Dieu ${ }^{34}$. La dévotion de Thomas envers Dieu était donc une dévotion amoureuse ${ }^{35}$. Transporté d'extase sous l'effet des prières et de la messe, le corps de Thomas se dissout littéralement et s'élève jusqu'à son bien-aimé.

33. Ystoria sancti Thome de Aquino de Guillaume de Tocco (1323), éd. C. Le Brun-Gouanvic, Toronto, 1996, p. 154-155: «Erat autem deuota supra modum eius oratio, qua sic liber in Deum deuotione mentis ascenderet, quasi nullus pondus carnis contrarium sustineret. Que consueta erat nunquam excitare motum rationi contrarium, qui corpus suum coegerat fieri sub ratione subiectum. Quod non solum ex dictis apparet que de eius humilitate, conuersatione et puritate sunt dicta, sed ex sequentibus, que sunt ueridica attestatione probata. Erat autem precipue deuotus ad sacrosanctum sacramentum altaris, de quo, quia concessum ei fuerat profundius scribere, donatum est ei deuotius celebrare. Cotidie enim unam missam dicebat, nisi eum infirmitas impedisset, et aliam audiebat socii uel alterius, ad quam ut frequentius ministrabat. Consueuerat autem frequentius rapi in missa tante diuotionis affectu ut totus perfunderetur lacrimis, qui tanti sacramenti sacris absorbebatur misteriis et reficiebatur ex donis. Vnde cum semel in conuentu Neapolitano in die dominice passionis, multis militibus astantibus, missam deuotius celebraret, in prosecutione cepti sacri misterii subito uisus est absortus sic ab altitudine sacramenti quasi diuinis uideretur admissus interesse misteriis et Christi hominis affici putaretur ex penis; quod indicare uidebatur diutina mentis abstractio et inundans perfusio lacrimarum. Quo diutius sic manente, admirati fratres accesserunt, et ipsum tangentes ut prosequeretur sacra misteria, a profunditate qua raptus uidebatur esse misterii excitauerunt.»

34. Ibid., cap. 11, p. 112.

35. Guillaume revient une autre fois sur ce point, voir ibid., cap. 16, p. 123: «Iste in sponsam Dei sapientiam habere meruit.» («Il méritait d'avoir la Sagesse de Dieu pour fiancée.») 
Pour autant, le corps joue un rôle: même s'il échappe à la chair, Thomas est submergé de larmes. Pourquoi des larmes? Dans sa Somme théologique, Thomas dit que les larmes sont souvent provoquées par la tristesse mais qu'elles peuvent également jaillir sous l'impulsion d'une sorte de sentiment de tendresse (affectus teneritudine ${ }^{36}$. En outre, Guillaume affirme que les larmes de Thomas, notamment lors de la messe, proviennent moins de sa personne au sens propre que de son identification au Christ. L'expérience extracorporelle de Thomas l'unit à la souffrance du Christ avec une telle intensité que ses propres larmes deviennent celles de son bien-aimé ${ }^{37}$. On a ici un parfait exemple d' «amour d'amitié».

Aussi Thomas lie-t-il étroitement les émotions au corps. Dans le même temps, son analyse de l'amour stipule qu'un dépassement du corps est possible. Son disciple Guillaume représente les émotions de son héros selon ces deux approches distinctes dans le même passage. D'une part, il veut montrer un Thomas impassible, triomphant de la chair. En ce sens, ailleurs dans son récit, il assure que Thomas était à ce point «absorbé» que souvent il ne ressentait aucune douleur alors que son corps était soumis à une expérience douloureuse ${ }^{38}$. Donc, il pouvait en effet arriver que son saint ne ressentît rien. Et cependant, d'autre part, Guillaume le présente submergé par les larmes, au moment précis où son transport aurait dû être entièrement spirituel.

Remigio de' Girolami, autre disciple de Thomas, parvient lui aussi à une conclusion semblable. Dans un sermon prononcé à l'occasion de la mort de Béatrice d'Anjou, il prêche sur la bonne façon d'exprimer son deuil. Remigio commence par reconnaître une forme d' «amour d'amitié» dans la vie conjugale, au sens où les émotions de l'un des deux époux se reflètent en miroir chez l'autre. Pour autant, Remigio met en garde: un époux ne doit pas être éploré outre mesure à la mort de son épouse. En particulier, il ne doit pas se lamenter avec excès (modicum plora) en versant des larmes, en gémissant ou en hurlant de douleur ${ }^{39}$. «Nous devrions rendre grâce à Dieu pour la mort d'une épouse»,

36. Thomas D'Aquin, Summa theologiae, II-II, q. 82, a. 4, ad 3.

37. La théologie du «sacrifice des larmes» par le croyant durant la messe fut d'abord énoncée par Grégoire le Grand puis développée durant la Réforme grégorienne, voir P. NAGY, « Larmes et eucharistie. Formes du sacrifice en Occident au Moyen Âge central », dans N. Bériou, B. CASEAU et D. Rigaux éd., Pratiques de l'eucharistie dans les Églises d'Orient et d'Occident (Antiquité et Moyen Âge), Paris, 2009, vol. II, p. 1073-1109.

38. Voir par exemple Ystoria sancti Thome de Aquino..., cap. 47, p. 182: «mentis abstractio». Voir B. H. Rosenwein, «The Mystical Skeleton in the Thomistic Closet: Aquinas's Impassibility», Journal of Medieval Religious Cultures, 36/2 (2010), p. 233-246.

39. Pour les textes de référence de Remigio de' Girolami, voir: Sermo 50, présenté en texte intégral (latin et traduction italienne) à http://www.e-theca.net/emiliopanella/remigio/8150.htm: «Sermone in morte di Beatrice d'Angiò. (Firenze 1315-1316)» et avec une explication sur les «pleurs excessifs», voir ID., Biblioteca Nazionale di Firenze, Conventi Soppressi D.1.937, 341v43r: «Plorare vero supra flere addit vocem. Ut dicatur plorare plus quam rorare, id est lacrimari vel 
écrit Remigio, «car même si le mariage est un bien, il est cependant préférable et plus bénéfique de vivre sans épouse qu'avec une épouse ${ }^{40}$. Ainsi, Remigio tente de réprimer les mouvements du corps, même s'il se délecte par ailleurs à décrire avec soin les larmes, les gémissements et les lamentations du deuil.

De toute évidence, la communauté émotionnelle de saint Thomas s'intéresse aux manifestations corporelles qui accompagnent les émotions. À la différence de la cour neustrienne, cette communauté émotionnelle rattache toutes les émotions au corps. Mais elle promulgue une hiérarchie des émotions et donc, par voie de conséquence, elle établit un ordre distinctif dans le champ des expériences corporelles. Au niveau inférieur, on compte les amours qui sont systématiquement ramenées à soi; ces amours-ci génèrent contractions et expansions, vociférations et sanglots. À un niveau plus élevé, on trouve les amours qui portent l'individu au-delà de lui-même; ces amours-là conduisent à l'extase qui néanmoins peut encore être enracinée dans la corporéité. Au niveau supérieur, on trouve les amours qui transportent au-delà des sens. Même là encore, une dernière marque corporelle demeure visible: ce sont les larmes seules, dissociées des cris, des sanglots ou de tout autre symptôme corporel. Mais nous allons voir qu' au $\mathrm{XIV}^{\mathrm{e}}$ siècle une autre communauté émotionnelle pouvait tolérer ces mêmes cris, voire les valoriser.

\section{Les larmes, les cris et les contorsions de douleur de Margery Kempe}

Margery Kempe (ca 1373-ca 1439) dut affronter «le mépris public, des menaces contre sa personne, [...] et même des enquêtes épiscopales visant la qualité de sa foi ${ }^{41}$. Au premier abord, on ne s'attend pas à la retrouver au cœur d'une communauté émotionnelle. Néanmoins son Livre - une autobiographie écrite à la troisième personne qui contient à peu près tout ce que nous savons

qui pluviam scilicet lacrimarum rarum facere scilicet interuptione clamose vocis et singultuum.» («Pleurer, c’est ajouter la voix aux larmes. Ainsi, on dit qu'une personne pleure plutôt qu'elle verse des larmes quand elle déverse un torrent de larmes entrecoupé par la clameur de ses cris et de ses hoquets.») Cité dans C. LAnsing, Passion and Order: Restraint of Grief in the Medieval Italian Communes, Ithaca, 2008, p. 57, n. 28.

40. Remigio de' Girolami, « Sermone in morte di Beatrice d'Angiò... » : «Regratiandum est Deo de morte coniugis, quia licet coniugium sit bonum tamen melius et salubrius est esse sine coniuge quam cum coniuge.»

41. The Book of Margery Kempe, éd. et trad. L. Staley, New York, 2001, p. vii. Pour le texte en vieil anglais, voir http://www.lib.rochester.edu/camelot/teams/tmsmenu.htm\#menu [The Book of Margery Kempe, L. Staley éd., Kalamazoo, 1996. Désormais «Le Livre en ligne »]. 
d'elle ${ }^{42}-$ ne détaille pas seulement l'opprobre public dont elle fut l'objet, mais également l'admiration d'un groupe de disciples.

Margery Kempe appartenait à une importante famille bourgeoise de la cité de Bishop's Lynn (aujourd'hui King's Lynn), où son père avait occupé à plusieurs reprises la fonction de maire (mayor) et plusieurs autres postes édilitaires ${ }^{43}$. À l'âge de vingt ans, elle épousa John Kempe, lui aussi bourgeois de Lynn ${ }^{44}$. Selon son propre témoignage, elle mit au monde quatorze enfants. Jamais elle ne renonça à son mariage pour rejoindre une communauté religieuse, même si par la suite elle et son mari firent vœu d'une vie chaste. Ils vécurent séparément un certain temps puis, lorsque son époux devint trop malade pour rester seul, elle reprit la vie commune afin de prendre soin de lui.

Margery Kempe avait le don d'irriter ses proches, les bourgeois et les paroissiens de Lynn, avec ses postures émotionnelles, en particulier ses irrépressibles sanglots. Contrairement à Marie d'Oignies qui avait obéi aux injonctions du prêtre qui lui demandait de contrôler ses larmes en se retirant à l'abri des regards ${ }^{45}$, Margery Kempe demeurait à la vue de tous même lorsqu'on lui demandait de se mettre à l'écart ${ }^{46}$. Elle accueillait comme un bienfait d'être soumise «aux calomnies et aux blasphèmes, aux rumeurs et blâmes du monde». Un tel traitement représentait «à ses yeux consolation et réconfort» ${ }^{47}$.

Méprisée par beaucoup, Margery Kempe était dans le même temps soutenue par «de nombreux et vénérables clercs, archevêques et évêques, docteurs en théologie, bacheliers. Elle s'entretint aussi avec un grand nombre d'ermites

42. Deux documents enregistrent l'entrée de Margeria/Margerie Kempe dans la Guilde de la Trinité de Lynn en 1437-1438. Voir The Book of Margery Kempe, éd. S. B. MeEch et H. E. Allen, Oxford, 1940, Appendice 3, p. 358-359.

43. Les éléments de preuve sont cités dans ibid., p. 359-362, montrant (p. 359) que «John Brunham [père de Margery] apparaît souvent et avec une visibilité croissante dans les archives de Lynn comme officier public (jurat, chambellan, membre du Parlement, maire, coroner, juge de paix, conseiller de la Guilde de la Trinité)».

44. Ibid.,p. 362-368: l'identification de John Kempe au mari de Margery dans la documentation est qualifiée de «probable».

45. JACQues DE Vitry, Vita B. Mariae Oigniacensis, Acta Sanctorum, Iun. IV., p. 640 : «Egressa clam ab ecclesia, in loco secreto $\&$ ab omnibus remoto se abscondit.» («Elle quitta l'église discrètement et se retira dans un lieu secret à l'abri des regards.»)

46. Même si elle essaie de rester silencieuse, voir Le Livre en ligne: «As sone as sche parceyvyd that sche schulde crye, sche wolde kepyn it in as mech as sche myth that the pepyl schulde not an herd it.» («Elle faisait tout pour étouffer ses cris, et que n'en soient pas incommodés ceux qui les entendaient.») Pour la traduction française, voir MARGERY KEMPE, Le Livre. Une mystique anglaise au temps de l'hérésie lollarde, trad. D. VIDAL (à partir de l'édition de S. B. Meech et H. E. Allen), Grenoble, 1987, p. 137. [Désormais abrégé en «Le Livre, trad. D. VIDAL».]

47. Le Livre, trad. D. VidaL, préambule, p. 47. Le Livre en ligne: «Sche was so usyd to be slawndred and repreved, to be cheden and rebuked of the world... that it was to her in a maner of solas and comfort». 
[...]. Et tous ceux à qui elle s'ouvrit de ses secrets dirent qu'il lui fallait aimer Notre Seigneur, pour la grâce qu'Il lui témoignait, lui conseillèrent de suivre ses penchants et inclinations, et d'avoir l'assurance qu'ils procédaient du SaintEsprit, non de l'esprit du mal ${ }^{48} \gg$.

Il apparaît donc que Margery Kempe n'appartenait pas à la communauté émotionnelle dominante de ses contemporains mais plutôt à une communauté marginale. Les normes émotionnelles de cette communauté n'en sont pas moins précieuses pour l'historien ${ }^{49}$. L'absence totale d'inhibition de Margery Kempe dans l'expression corporelle de l'émotion indique que nous sommes devant le modèle le plus radical de connexion entre le corps et les émotions parmi les exemples envisagés ici.

Le «texte représentatif» que nous avons choisi est un extrait du Livre de Margery qui relate ses expériences dans l'église du Saint-Sépulcre à Jérusalem:

Les moines levèrent alors une croix et guidèrent les pèlerins de l'un à l'autre lieu où notre Seigneur avait souffert son martyre et sa Passion, chacun, homme et femme, portant un cierge à la main. Et les moines toujours, tandis qu'ils avançaient, leur disaient ce qu'en chaque lieu notre Seigneur avait souffert. La créature [Margery] pleurait et sanglotait avec autant de violence que si elle avait vu de ses yeux de chair notre Seigneur souffrant à l'instant même de la Passion. Devant elle, en son âme, elle le vit en contemplation réellement, et fut envahie de pitié. Quand ils gravirent le Mont du Calvaire, elle s'écroula, incapable de rester debout ni de s'agenouiller. Elle roula à terre, corps disloqué, bras étalés, poussant des hurlements terribles, comme si son cœur s'était brisé. Car dans la cité de son âme, elle voyait cette réalité toute sensible: notre Seigneur mis en croix. Sous ses yeux elle entendait et voyait, de vue spirituelle, notre Dame en affliction, et saint Jean, Marie-Madeleine, et tant d'autres, qui aimèrent notre Seigneur.

Tant de pitié elle éprouva, tant de douleur, en voyant les tourments de notre Seigneur, qu'elle ne pouvait s'empêcher de crier et de hurler, dût-elle en mourir. Et ce fut là le premier cri qu'elle eût jamais crié en contemplation. Et cette sorte de cri dura longtemps après. Jamais personne n'eut ainsi pu crier. Elle en souffrit mépris et blâme, grandement. Si terribles et prodigieux ses cris, que l'on en fut

48. Le Livre, trad. D. VIDAL, préambule, p. 48. Le Livre en ligne: «many worshepful clerkys, bothe archebysshopys and bysshoppys, doctowrs of dyvynyté and bachelers also. Sche spak also wyth many ankrys... And thei alle that sche schewed hyr secretys unto seyd sche was mech bownde to loven ower Lord for the grace that he schewyd unto hyr and cownseld hyr to folwyn hyr mevynggys and hyr steringgys and trustly belevyn it weren of the Holy Gost and of noon evyl spyryt.»

49. Concernant d'autres membres probables de cette même communauté, voir en particulier D. S. Ellis, «Margery Kempe and King's Lynn», dans S. J. McEntire éd., Margery Kempe : A Book of Essays, New York, 1992, p. 139-163, et J. WiLson, «Communities of Dissent: The Secular and Ecclesiastical Communities of Margery Kempe's Book», dans D. WATT éd., Medieval Women in their Communities, Toronto, 1997, p. 155-185. 
épouvanté, hormis ceux qui les avaient déjà entendus et savaient la raison. Et si souvent elle en poussait, que son corps en était accablé de faiblesse ${ }^{50}$.

On voit que chaque ressenti de Margery s'accompagne de mouvements corporels extravagants. Lorsqu'elle entend les frères évoquer le martyre du Christ, elle éclate en sanglots et crie. À la vue du Golgotha, elle tombe à terre et se tord de douleur, hurle, sent son cœur pour ainsi dire éclater. Comme elle contemple en pensée la douleur de ceux qui assistèrent à la Passion du Christ ainsi que ses propres souffrances, elle n'éprouve pas seulement à son tour douleur et compassion, elle se met à pleurer et à hurler. Ces spasmes semblent s'être calmés au bout d'un certain temps, il reste qu'une décennie durant Margery Kempe connaîtra ces crises de hurlements qui étaient accompagnées d'une sensation envahissante de faiblesse corporelle ${ }^{51}$.

Le corps émotionnel de Margery Kempe est tellement excessif qu'on n'a pas manqué d'en faire une malade psychotique, épileptique ou hystérique ${ }^{52}$. Cependant, Emily Huber, parmi d'autres, insiste pour rappeler que l'affectivité de Margery n'était guère différente de celle d'autres mystiques et ascètes de son temps ${ }^{53}$. De plus, comme je l'ai dit, elle n'était pas dépourvue de soutiens

50. Le Livre, trad. D. VIDAL, I, ch. 28, p. 136-137. Le Livre en ligne: «Than the frerys lyftyd up a cros and led the pylgrimys abowte fro on place to an other wher owyr Lord had sufferyd hys peynys and hys passyons, every man and woman beryng a wax candel in her hand. And the frerys alwey, as thei went abowte, teld hem what owyr Lord sufferyd in every place. And the forseyd creatur wept and sobbyd so plentyuowsly as thow sche had seyn owyr Lordwyth hir bodyly ey sufferyng hys Passyon at that tyme. Befor hir in hyr sowle sche saw hym veryly be contemplacyon, and that cawsyd hir to have compassyon. And whan thei cam up onto the Mownt of Calvarye sche fel down that sche mygth not stondyn ne knelyn but walwyd and wrestyd wyth hir body, spredyng hlr armys abrode, and cryed wyth a lowde voys as thow hir hert schulde a brostyn asundyr, for in the cité of hir sowle sche saw veryly and freschly how owyr Lord was crucifyed. Beforn hir face sche herd and saw in hir gostly sygth the mornyng of owyr Lady, of Sen John and Mary Mawdelyn, and of many other that lovyd owyr Lord. And sche had so gret compassyon and so gret peyn to se owyr Lordys peyn that sche myt not kepe hirself fro krying and roryng thow sche schuld a be ded therfor. And this was the fyrst cry that evyr sche cryed in any contemplacyon. And this maner of crying enduryd many yerys aftyr this tyme for owt that any man myt do, and therfor sufferyd sche mych despyte and mech reprefe. The cryeng was so lowde and so wondyrful that it made the pepyl astoynd les than thei had herd it beforn and er ellys that thei knew the cawse of the crying. And sche had hem so oftyntymes that thei madyn hir ryth weyke in hir bodyly myghtys.»

51. Le Livre, trad. D. VidAL, I, ch. 57, p. 233 : «Ces hurlements durèrent dix ans ». Le Livre en ligne: «And this maner of crying enduryd the terme of ten yer.»

52. La littérature sur ces diagnostics est citée par E. R. HubER, «For Y am sorwe, and sorwe ys $\mathrm{Y} »$, Melancholy, Despair, and Pathology in Middle English Literature (Ph. D. diss., University of Rochester, 2008), p. 176-179.

53. Ibid. En complément, voir C. W. Bynum, «The Female Body and Religious Practice in the Later Middle Ages », dans M. FeHER avec R. NAdDafF et N. TAZI éd., Fragments for a History of the Human Body, Part One, New York, 1989, p. 161-219. C. W. Bynum souligne (p. 162) que 
ni d'admirateurs. Même si nous n'entendons pas leurs propres cris, on peut considérer qu'ils partageaient sa façon de considérer si intensément les souffrances du Christ tout comme ils adhéraient aux modes d'expression de sa douleur et de sa compassion. Cette voie n'est pas sans lien avec les vues d'un Thomas d'Aquin sur les rapports corps/âme, mais on ne retrouve rien ici de l'ambivalence de la communauté de Thomas concernant la participation de la chair. Margery ne recherchait pas ces spasmes et ces crises de larmes qui l'accablaient «sans que personne ne pût rien y faire ${ }^{54}$. Pour autant, ces moments lui étaient doux : «Jamais ses cris n'advenaient sans qu'elle eût connu dévotion très douce et profonde contemplation ${ }^{55}$.» Même l'opprobre public lui était doux : «C'était à ses yeux quelque consolation et réconfort, que de souffrir ces maux pour l'amour de $\mathrm{Dieu}^{56}$.» Ainsi, c'est l'intégralité de la séquence qui est approuvée, à la fois intellectuellement et émotionnellement, par Margery mais aussi - et je plaide en ce sens - par sa communauté émotionnelle, depuis le sentiment intérieur amour de Dieu, compassion - jusqu'aux manifestations extérieures - hurlements, rejet social.

\section{Contextes}

Jusqu'ici nous avons laissé de côté le contexte d'ensemble de ces trois communautés émotionnelles. Bien entendu, ces contextes sont cruciaux si nous voulons apprécier à leur juste place ces trois exemples. Il ne sera possible ici que d'esquisser la discussion.

Les communautés émotionnelles existent à l'intérieur de cadres plus vastes, dans des sociétés où plusieurs d'entre elles, voire un grand nombre, peuvent cohabiter. Il convient d'imaginer alors un grand cercle à l'intérieur duquel sont disposés des cercles plus petits: certains d'entre eux se recoupent partiellement, d'autres mordent quelque peu à l'extérieur du cercle le plus

«la spiritualité féminine durant [la période 1200-1500] a été particulièrement somatique». Il est probable que l'exhibitionnisme émotionnel de Margery Kempe ait été plus mal vu à son époque que précédemment en raison de la suspicion croissante de la part des hommes d'Église pour qui ce genre de comportement était l'indice d'une possession démoniaque. Voir N. CACIOLA, Discerning Spirits : Divine and Demonic Possession in the Middle Ages, Ithaca, 2003. D'ailleurs, Margery fut accusée d'être lollarde.

54. Le Livre, trad. D. VIDAL, I, ch. 28, p. 136. Traduction modifiée. Le Livre en ligne: «for owt that any man myt do.»

55. Le Livre, trad. D. Vidal, I, ch. 28, p. 137. Le Livre en ligne: «And thei come nevyr wythowtyn passyng gret swetnesse of devocyon and hey contemplacyon.»

56. Le Livre, trad. D. VIDAL, préambule, p. 47. Le Livre en ligne (après l'évocation de réprimandes publiques): «It was to her in a maner of solas and comfort whan sche sufferyd any dysese for the lofe of God.» 
important. Pour la période que nous avons considérée ici, le cercle le plus étendu représente les valeurs et les normes de l'Occident chrétien dans leur évolution, qui prend la forme d'une «Chrétienté» consciente d'elle-même à partir du XII ${ }^{\mathrm{e}}$ siècle ${ }^{57}$. À l'intérieur de ce cercle étendu, on retrouve les groupes que nous avons pris en compte ici: les hommes de foi de la cour neustrienne, des prêtres dominicains, une mystique laïque et ses soutiens. En revanche, le cercle des communautés juives par exemple devrait être placé partiellement à l'extérieur de l'ensemble le plus vaste ${ }^{58}$. Les communautés paysannes également se retrouveraient probablement en partie au-delà de cette circonférence ${ }^{59}$, tandis que la majeure partie de la communauté (ou des communautés) seigneuriale(s) serait incluse ${ }^{60}$. On pourrait envisager de la même façon les émotions «au cœur de la cité ${ }^{61}$.

Les exemples abordés ici sont loin d'épuiser la diversité des communautés émotionnelles, y compris pour chacune des périodes considérées. Nous pouvons entrevoir (très succinctement en raison de la rareté des sources) une deuxième

57. Sur les frontières mentales de cette Chrétienté, voir D. Iogna-Prat, Ordonner et exclure. Cluny et la société chrétienne face à l'hérésie, au judaïsme et à l'islam (1000-1150), Paris, 2000².

58. Partiellement, mais non en totalité. Sur l'influence de certains aspects de la culture de la Chrétienté sur les communautés juives, voir I. G. MARCus, Rituals of Chidlhood: Jewish Acculturation in Medieval Europe, New Haven, 1996; L. Garcia-Ballester, L. Ferre et E. Feliu, «Jewish Appreciation of Fourteenth-Century Scholastic Medicine», Osiris, $2^{\text {de }}$ sér. 6 (1990), p. 85-117; E. S. Horowitz, Reckless Rites : Purim and the Legacy of Jewish Violence, Princeton, 2006. Voir aussi S. Einbinder, compte rendu de I. Yuval, Two Nations in Your Womb: Perceptions of Jews and Christians in Late Antiquity and the Middle Ages, Speculum, 82/3 (2007), p. 780-781: «Une vue plus large doit [...] tenir compte des moments de synthèse et de production culturelle [commune], des moments d'interaction quotidienne, même furtifs, entre voisins, clients, et peut-être amis ». Sur ce sujet, voir F. H. Mitilineos, English Convivencia: Aspects of Christian-Jewish Cooperation in Medieval England, 1189-1290, Ph.D. diss., Loyola University Chicago, 2009. Je remercie Susan Einbinder de m'avoir signalé la plupart de ces références.

59. P. Freedman, «Peasant Anger in the Late Middle Ages», dans B. H. Rosenwein éd., Anger's Past: The Social Uses of an Emotion in the Middle Ages, Ithaca, 1998, chap. 8, montre bien que nos connaissances sur les émotions paysannes découlent largement des représentations de l'élite. Là où elles ont été directement exprimées, ce fut en termes pieux, parfaitement en conformité avec les normes du cercle de la Chrétienté.

60. Voir par exemple S. D. White, «The Politics of Anger», dans Anger's Past..., chap. 6, qui montre que les normes de la colère, et des émotions afférentes, sont assez distinctes (bien que pas complètement) dans les cercles seigneuriaux laïcs des normes de l'élite religieuse à la même période. D'un autre côté, les conceptions de l'amour sont très proches, voir C. BALADIER, Érôs au Moyen Âge. Amour, désir et «delectatio morosa », Paris, 1999.

61. Voir par exemple les considérations sur les émotions des Brugeois au milieu du $\mathrm{XV}^{\mathrm{e}}$ siècle dans J. Dumolyn et É. LeCuPPRe-Desjardin, « Propagande et sensibilité : la fibre émotionnelle au cœur des luttes politiques et sociales dans les villes des anciens Pays-Bas bourguignons. L'exemple de la révolte brugeoise de 1436-1438 », dans É. Lecuppre-Desjardin et A.-L. VAn Bruaene éd., Emotions in the Heart of the City (14th-16th century), Turnhout, 2005, p. 41-62. 
communauté émotionnelle au $\mathrm{VII}^{\mathrm{e}}$ siècle dans la lettre de Bertegisèle qui use explicitement d'expressions d'affection et d'amour ${ }^{62}$. Il est plus aisé d'identifier des groupes alternatifs pour le XIII ${ }^{\mathrm{e}}$ siècle où l'état des sources est nettement plus favorable. Là, à l'intérieur du cercle dominant de la Chrétienté, on peut observer une rivalité entre les dominicains et les franciscains qui valorisent fortement une dévotion charnelle ${ }^{63}$, ou encore les mystiques dont les extases sont rendues par des métaphores physiques et érotiques ${ }^{64}$. Les normes émotionnelles et corporelles qui prévalent chez Margery Kempe reprennent les modèles d'abord élaborés par les franciscains et les mystiques mais elles font également écho à la théâtralisation de la vie politique au $\mathrm{Xv}^{\mathrm{e}}$ siècle qui est un élément de contexte important à prendre en compte ${ }^{65}$.

Les évolutions d'une communauté émotionnelle à l'autre que nous avons suivies dans cet article montrent une orientation, depuis le refus, parfois dédaigneux, d'une expression émotionnelle qui passe par le corps, vers une timide acceptation, jusqu'à une intégration pleine et entière. Doit-on y voir un effet propre aux groupes particuliers qui ont été considérés ici, ou bien cette trame renvoie-t-elle à un phénomène de plus grande ampleur? Cela semble être le cas. Ce n'est pas pour autant révélateur d'un «progrès» en marche dans l'histoire de l'Occident, depuis un rejet du corps vers son intégration enthousiaste. À l'époque victorienne par exemple, on le sait, le corps sera de nouveau rejeté66. L'orientation perceptible dans les sources médiévales s'explique assurément par tout un ensemble de facteurs. Je n'en citerai qu'un: l'influence grandissante des couches bourgeoises et de leurs normes. Les pratiques émotionnelles et

62. Voir supra, n. 15.

63. Voir Thomas de Celano, Vita prima S. Francisci Assisiensis, S. Bonaventure College éd., Florence, 1926, II, III, 94, p. 102, où François, lors de la vision au mont Alverne, se sent tour à tour triste (tristis) et gai (laetus), avant de voir les marques des clous de la crucifixion apparaître sur ses mains et ses pieds.

64. Voir l'étude de référence de C. W. Bynum, Holy Feast and Holy Fast: The Religious Significance of Food to Medieval Women, Berkeley, 1987. Depuis, la littérature sur les rapports entre corps et religion a prospéré. La problématique du corps et des émotions est distincte mais les deux sujets demeurent étroitement liés.

65. Voir P. Arnade, Realms of Ritual : Burgundian Ceremony and Civic Life in Late Medieval Ghent, Ithaca, 1996; D. Nicholas, «In the Pit of the Burgundian Theater State: Urban Traditions and Princely Ambitions in Ghent, 1360-1420», dans B. A. Hanawalt et K. L. Reyerson éd., City and Spectacle in Medieval Europe, Minneapolis-Londres, 1994, p. 271-295; K. M. PhiLliPs, «The Invisible Man: Body and Ritual in a Fifteenth-Century Noble Household», Journal of Medieval History, 31 (2005), p. 143-162.

66. Voir P. N. Stearns, American Cool : Constructing a Twentieth-Century Emotional Style, New York, 1994,p. 66: «[À l’ère victorienne], les représentations nouvelles du corps avaient séparé les émotions de leur fonction somatique bien plus qu'elles ne l'avaient été dans les conceptions traditionnelles et qu'elles ne le seraient de nouveau au Xx ${ }^{\mathrm{e}}$ siècle »; voir aussi plus loin, p. 67-68. 
dévotionnelles de la classe bourgeoise valorisent le corps dans la relation sociale. Le père de saint François était un marchand, les mystiques béguines étaient issues des milieux urbains du nord de l'Europe, Margery Kempe appartenait à une famille bourgeoise.

Cela dit, Margery Kempe dut également affronter le mépris de certains membres de son milieu. Cela remet-il en cause pour autant l'idée que les valeurs bourgeoises s'imposent? Il est important de rappeler qu'on ne saurait envisager l'existence d'une seule communauté émotionnelle bourgeoise. Il faudrait sans doute en concevoir plusieurs, lesquelles auraient néanmoins certaines caractéristiques communes. En outre, les normes tendent également à évoluer à mesure que la théorie médicale commence à dominer les conceptions des passions ${ }^{67}$. De même, l'Église en vient peu à peu à reconnaître une inspiration démoniaque plutôt que divine dans la mystique féminine ${ }^{68}$. Également, une expression émotionnelle plus intime se développe dans certains groupes issus des milieux urbains, comme on le voit par exemple dans la devotio moderna ${ }^{69}$. $\mathrm{Au} \mathrm{XVI} \mathrm{I}^{\mathrm{e}}$ siècle, le corps sera un corps médicalisé, perçu non comme il l'est de nos jours, à savoir un site de consommation et de production ${ }^{70}$, mais plutôt comme un chaudron d'esprits et d'humeurs. Ainsi, on entrevoit encore d'autres modes de l'incorporation de l'émotion qui se démarquent de la corporéité médiévale même s'ils prennent appui sur celle-ci.

Quelle est la signification de ces différences dans l'usage et la conception du corps pour l'expérience de l'émotion ? Comment appréhender la vie émotionnelle d'une communauté en sachant que les hommes de la cour de Neustrie valorisaient une dissociation du corps et de l'émotion, que les disciples de Thomas d'Aquin faisaient une place même timide au corps, ou que Margery Kempe puisait dans ses cris et ses larmes intarissables un sentiment de douceur? Ressentaient-ils tous les mêmes émotions universelles? On peut en douter fortement. Les émotions ne sont pas des entités objectives qui ne feraient que se manifester de diverses façons suivant la diversité des lieux mais elles sont le fruit d'évaluations socialement déterminées. Ces évaluations façonnent les émotions, leurs modalités d'expression mais aussi - je plaide en ce sens - la

67. Voir P. BASEOTTO, «Exploring the Humoral Self: Elizabethan and Early Stuart Churchmen and Galenic Medicine», dans Perspectives on the Language and Culture of Science, à paraître.

68. Voir D. ElLiotT, Proving Women: Female Spirituality and Inquisitional Culture in the Later Middle Ages, Princeton, 2004; N. CACIOLA, Discerning Spirits...

69. Voir J. VAn Engen, Sisters and Brothers of the Common Life: The Devotio Moderna and the World of the Later Middle Ages, Philadelphie, 2008.

70. Voir O. E. Dror, «Creating the Emotional Body: Confusion, Possibilities, and Knowledge», dans P. N. Stearns et J. Lewis éd., An Emotional History of the United States, New York, 1998, chap. 9 . 
manière dont elles sont ressenties. L'amour devait être ressenti différemment lorsque ses manifestations corporelles étaient empêchées et dévalorisées ou bien lorsqu'il était vécu comme transcendant le corps. Et il devait être vécu encore différemment lorsque ce même amour produisait hurlements et spasmes corporels. J'espère avoir montré dans cet article que la relation entre l'émotion et le corps n'est ni obligatoire ni invariable mais qu'elle dépend des normes et des besoins en matière d'expression à l'intérieur de chaque communauté émotionnelle.

Barbara H. Rosenwein - Loyola University, Department of History, 1032 W. Sheridan Road, Chicago, IL 60660, États-Unis

\section{Les communautés émotionnelles et le corps}

Il y a longtemps Alain de Lille affirmait que les gestes et les expressions faciales des émotions étaient des fenêtres ouvertes sur la «disposition intérieure» d'une personne, tandis que de nos jours Paul Ekman soutient que certaines expressions faciales constituent des «émotions basiques». Cependant, comme cet article le montre, en réalité le lien entre le corps et les émotions est (et demeure) extrêmement divers. L'article aborde les émotions dans le contexte des «communautés émotionnelles» - des groupes sociaux à l'intérieur desquels les individus sont animés par des intérêts, des valeurs et des styles émotionnels communs ou semblables. Les communautés émotionnelles intègrent (ou n'intègrent pas) le corps dans l'expression de l'émotion de différentes manières. Ce constat est illustré par trois exemples médiévaux. À la cour mérovingienne de Neustrie au VII siècle, les émotions étaient rarement incarnées. Au contraire, pour Thomas d'Aquin et ses disciples au XIII siècle, les émotions étaient explicitement associées au corps et aux changements somatiques. Mais cependant, pour ce groupe de dominicains, les émotions pouvaient, si elles étaient orientées de façon appropriée, transcender le corps. Enfin, Margery Kempe et ses semblables usaient de leur corps en manifestant leurs émotions de façon dramatique, par des hurlements, des larmes ou des contorsions de douleur. L'article se clôt en suggérant que ces variations dans les modalités d'incorporation permettent de questionner la conception d'émotions «universelles».

Communautés émotionnelles - Margery Kempe - Mérovingiens - Neustrie Thomas d'Aquin. 


\section{Emotional Communities and the Body}

Alain de Lille long ago proclaimed that gestures and facial expressions were windows onto a person's «internal disposition», while today Paul Ekman claims that certain facial expressions represent «basic emotions». However, as this paper argues, in fact the association between the body and emotion is (and remains) highly variable. The paper treats emotions within the context of «emotional communities» - social groups within which people are animated by common or similar interests, values, and emotional styles. Emotional communities have different ways of incorporating (or not) the body in emotional expression. This point is exemplified by three medieval examples. At the seventh-century Merovingian court in Neustria, emotions were rarely embodied. By contrast, for Thomas Aquinas and his disciples in the thirteenth century, emotions were clearly associated with the body and somatic changes. Even so, for this group of Dominicans, emotions could, if properly directed, transcend the body. Finally, Margery Kempe and others of her ilk employed their bodies in dramatic expressions of feeling via roars, tears, and writhing. The paper ends by suggesting that these variations in embodiment help challenge the view of «universal» emotions.

Emotional communities - Margery Kempe - Merovingians - Neustria - Thomas Aquinas. 
